\title{
Treatment planning for the layer-stacking irradiation system for 3D conformal heavy-ion radiotherapy
}

Nobuyuki Kanematsu, Masahiro Endo, Yasuyuki Futami, ${ }^{a}$ and Tatsuaki Kanai

Department of Medical Physics, Research Center for Charged Particle Therapy,

National Institute of Radiological Sciences, 4-9-1 Anagawa, Inage-Ku, Chiba 263-8555,

Japan

Hiroshi Asakura

Accelerator Engineering Co., 2-13-1 Konakadai, Inage-Ku, Chiba 263-0043, Japan

Hiroyoshi Oka

Asahi-Kasei Jyoho System Co., Ltd., 2-10-14 Ryogoku, Sumida-Ku, Tokyo 130-0026,

Japan

a Present address: Department of Proton Beam Therapy, Shizuoka Cancer Center, 1007 Shimonagakubo,

Nagaizumi-Chou, Suntou-Gun, Shizuoka 411-8777, Japan 


\section{Ken Yusa}

Japan Science and Technology Corporation, 4-1-8 Honcho, Kawaguchi, Saitama 332-0012, Japan

We have upgraded a heavy-ion radiotherapy treatment-planning system to adapt for the layer-stacking irradiation method, which is to conform a variable SOBP to a target volume by means of dynamic control of the conventional beam-modifying devices. The biophysical model, the beam-setup logic, and the dose-calculation algorithm implemented for the layer-stacking method are described and the expected clinical usability is discussed.

The layer-stacking method was integrated in perfect accordance with the ongoing conventional treatments so that the established protocols, which are the clinically optimized dose fractionation schemes, will still be valid. On the other hand, a simulation study indicated a substantial improvement of dose distribution with the 
layer-stacking method though the significance may depend on the size, shape, and location of the tumor. The completed treatment system will provide an option for improved conformal radiotherapy without interfering with the conventional method and we expect a gradual expansion of the clinical cases applicable to the layer-stacking method.

Key words: heavy-ion radiotherapy, 3D conformal radiotherapy, dynamic multileaf collimation, spread-out Bragg peak, linear-quadratic model 87.53.Tf, 87.53.Kn, 87.53.Ln

\section{INTRODUCTION}

The advantages of heavy-ion beams for radiotherapy are mainly owing to excellent physical properties and a concurrent radiobiological enhancement. A heavy-ion beam 
can be precisely controlled to administrate the maximum dose at a tumor site, where the relative biological effectiveness (RBE) will be also at maximum, while a proton beam is usually modeled as a low-LET radiation with constant RBE between 1.0 and 1.1. Even though complexities and uncertainties in the fragmentation processes and in the biological responses for high LET have not been fully resolved yet, the large RBE at Bragg peak and the small lateral penumbra would be advantages of heavy-ion radiotherapy over proton or lighter particles.

Clinical studies on carbon-ion radiotherapy have been carried out at National Institute of Radiological Sciences (NIRS) in Japan since 1994, using medical accelerator complex HIMAC. ${ }^{1}$ Over one thousand patients have been treated so far, ${ }^{2}$ where applied was the conventional irradiation method summarized as follows: ${ }^{3}$ A treatment beam is laterally broadened by a wobbler-scatterer system. A range-modulating device called a ridge filter produces a spread-out Bragg peak (SOBP), which is a depth span of a constant biologically effective dose, to cover the maximum width of the target. A range compensator adjusts the distal surface of the SOBP volume to match the distal surface 
of the target volume together with a range shifter. Either a multileaf collimator (MLC) or a patient collimator defines the beam field covering the outer contour of the projected target. However, since the width of an actual target varies within the field, the fixed SOBP produced by a ridge filter results in undesirable dosage to the normal tissue in front of the target.

The layer-stacking irradiation method was proposed to resolve this problem, ${ }^{4}$ and the HIMAC beam delivery system was upgraded to put this technique into practice. $^{5}$ Instead of conventional ridge filters, a single filter will be used to spread the Bragg peak to the size of several $\mathrm{mm}$ in water. ${ }^{6}$ The resultant small spread-out Bragg peak (minipeak) will be longitudinally scanned over the target volume in a stepwise manner. The target volume is longitudinally divided into slices, to each of which the minipeak is conformed using the MLC and the range shifter. A sequence of the irradiation steps with appropriate dose weights produces SOBP large enough to cover the target while trimming out the undesirable irradiations in front of the thin part of the target. Consequently, a variable SOBP coinciding to the target volume will be formed. The 
data format for the interface between the treatment planning system and the beam

delivery system has been upgraded to describe sequences of irradiation steps, which should be determined by the treatment planning system.

The layer-stacking method may be positioned in between the conventional method and the spot-scanning method. ${ }^{7}$ In recent years, the spot-scanning method for particle radiotherapy has been practiced in Switzerland, ${ }^{8}$ and in Germany. ${ }^{9}$ The spot-scanning method has a potential to deliver truly optimum $3 \mathrm{D}$ dose distribution, ${ }^{10}$ but, at the same time, it requires a precise and complicated beam delivery system. The spot-scanning system for HIMAC should be practical only by an addition of a dedicated beam line, for which efforts are currently being made. ${ }^{11}$ On the other hand, this approach is to seamlessly improve the quality of treatment by means of an upgrade of the conventional treatment system without interfering with the ongoing clinical programs currently with over two hundred patients per year. 
As a natural consequence of this approach, the layer-stacking method should be practiced in the scope of the current treatment protocols. Therefore, we decided not to refine the present theoretical framework, which the protocols are based on, at this point of time even though it may be too primitive and outdated compared to the recent studies on heavy-ion radiotherapy treatment planning. ${ }^{12} 13 \quad 14$ In this paper, we describe the modifications made to the existing treatment planning system for HIMAC, ${ }^{15}$ and we discuss the clinical usability of this irradiation method.

\section{MATERIALS AND METHODS}

\section{Biophysical modeling}

For the conventional method, the biophysical modeling was not in the scope of treatment planning but was included in ridge-filter design and thus the treatment planning system only handles the resultant data tables of the depth-dose curves and the 
biological effects of the range-modulated clinical beams. For the layer-stacking method, however, the range modulation is dynamically designed in treatment planning, for which we employed the same biophysical model based on the linear-quadratic (LQ) model as that for the conventional ridge filter design: ${ }^{16}$

(1) The cell survival responses for carbon-ion radiation were extensively measured, ${ }^{17}$ and the LQ model parameters $\alpha$ and $\beta$ were obtained as a function of LET, where human salivary grand (HSG) tumor cell line was chosen to represent all the human cells because its response was found to be in the middle of a variety of biological species. ${ }^{18}$ Figs. 1(a) and 1(b) show the adopted LQ parameters $\alpha$ and $\beta$ as a function of LET.

(2) The doses and dose-averaged LET's for the unmodulated beams were analytically calculated considering range straggling and projectile fragmentations based on the empirical model. ${ }^{19}$ Figs. 1(c) and 1(d) show the calculated dose and the dose-averaged LET for the unmodulated clinical beams of energies 290, 350, and $400 \mathrm{MeV}$ per nucleon as a function of "beam depth" measured from the beam source. The LQ 
parameters can then be related to the beam depth through the LET, ignoring ion species

of the fragments and approximating the monochromatic LET by the dose-averaged

LET.

(3) The minipeak dose $D_{G}$ was obtained by the convoluting the effect of the minipeak

filter to the unmodulated dose $D_{l}$ and the effective LQ parameters $\alpha_{G}$ and $\beta_{G}$ for the

minipeak beam were calculated by dose averages; ${ }^{20}$

$D_{G}(z)=\int D_{1}(z+t) G(t) d t$

$\alpha_{G}(z)=\frac{\int \alpha(z+t) D_{1}(z+t) G(t) d t}{D_{G}(z)}$,

$\sqrt{\beta_{G}(z)}=\frac{\int \sqrt{\beta(z+t)} D_{1}(z+t) G(t) d t}{D_{G}(z)}$,

where, $G(t)$ is the pseudo-Gaussian probability function with mean $5.4 \mathrm{~mm}$ and standard deviation $2.5 \mathrm{~mm}$ for the distribution of the minipeak filter water-equivalent thickness $t$. 
Fig. 2(a) shows the calculated minipeak doses and the corresponding measured doses.

The measured doses in the plateau region were higher than the calculated counterparts were, implying the incompleteness of the physical model in the calculation. We have therefore adopted the measured depth-dose curves in the subsequent calculations. In other words, the physical dose distributions are not part of the hypothetical biophysical model.

(4) On the other hand, the calculation errors of the LET's and the resultant RBE's are ignored, since they are part of the adopted biophysical model. An RBE is in general a conversion factor from a physical dose to the ${ }^{60} \mathrm{Co}$ dose for the same survival level, which was, however, too abstract for the conventional method, where the survival level had to be fixed for designing ridge filters. The clinical RBE was therefore defined as the RBE value at $10 \%$ survival level in the in vitro cell experiments, ${ }^{17}$ multiplied by the clinical factor to correct the effectiveness to that for in vivo. The clinical RBE of the minipeak as a function of depth is then formalized by; 


$$
R B E_{G}(z)=\frac{1.43 \cdot 4.035 \cdot 2 \beta_{G}(z)}{\sqrt{\alpha_{G}(z)^{2}-4 \beta_{G}(z) \ln 0.1-\alpha_{G}(z)}},
$$

where 4.035 Gy is the experimentally obtained $10 \%$-survival ${ }^{60} \mathrm{Co}$ dose for the HSG cells and survival level 10\%, corresponding to clinical dose of $5.77 \mathrm{GyE}$, was taken for the typical value for one fraction. Clinical factor 1.43 was originally to account for the difference between in vivo and in vitro but, in reality, it was introduced to preserve the GyE dose scale of the historical fast neutron radiotherapy at NIRS. ${ }^{18}$

(5) The clinical dose in unit of GyE from the minipeak beam, $C_{G}$, is defined simply by product;

$$
C_{G}(z)=R B E_{G}(z) D_{G}(z)
$$

In this model, the RBE is dose-independent because it was defined at the fixed survival level irrelevantly to the actual cell survival to be caused by the irradiation. Figs. 2(b) 
and 2(c) show the clinical RBE's and the clinical doses, respectively, for the minipeak beams.

\section{Range adjustment and field delineation}

The layer-stacking method and the conventional method share many features in the beam setup, such as the analysis of target depths using CT images, the choice of the optimum beam energy and wobbling condition, and the design of a range compensator. The essential difference lies in the stepwise control of the range shifter and MLC to conform SOBP to the target volume including the specified longitudinal and lateral margins.

Fig. 3 shows an example of a spherical target, where the smooth line represents the cross section area of the target as a function of "compensated patient depth" measured from the entrance of the range compensator, with which the distal surface of the target is 
fully equalized to the maximum depth. The partially numbered boxes represent the slices of the target. We chose the constant slice width of $2.5 \mathrm{~mm}$ in water to smoothly form a large SOBP.

The beam range in a patient for each step is adjusted by the range shifter in such a way that the peak of the physical minipeak curve be located at the center of the corresponding target slice. Similarly, the field for each step is conformed by MLC to the corresponding target slice with a few constraints: (1) The opening between pairing left and right leaves below $10 \mathrm{~mm}$ is forced to $10 \mathrm{~mm}$ if not completely closed. (2) In a case where MLC field area would be smaller than $400 \mathrm{~mm}^{2}$, the MLC setup for the adjacent deeper slice is applied instead. (3) The MLC setup for the deepest slice, namely the largest field, is applied to the nearby slices within $30 \mathrm{~mm}$ in water. Constraints (1) and (2) are due to the limitation of the employed broad-beam dose calculation algorithm that is not capable to precisely handle a narrow beam while constraint (3) is to limit the variable SOBP to be at least $30 \mathrm{~mm}$ in water because it takes finite number of minipeaks to form a sufficient dose level. 


\section{Step-weight optimization}

The objective for the step-dose weighting is to prescribe the specified nominal clinical dose to the given target volume as uniformly as possible. However, it is in principle impossible to achieve perfect uniformity over the variable-SOBP volume since the moving MLC partially blocks the fragmentation tail after the Bragg peak, which is the contribution of light ions originated in projectile fragmentations in flight. We therefore employed an iterative approach to determine the optimum dose weights for the steps. ${ }^{21}$

The irradiation steps and the corresponding target slices are numbered with index $i$ from the distal end $(i=1)$ to the proximal end $(i=N)$. We take $2 N-1$ equally spaced samples of compensated patient depths with index $j$. As shown in Fig. 4 , depth $j=1$ is at the peak of the clinical dose from step $i=1$ and depth $j=2 N-1$ is at the peak of the clinical dose from step $i=N$. The clinical dose at depth $j$ from step $i$ is given by; 
$C_{G i j}=C_{G}\left(z_{G}+T_{i}-T_{1}+\delta_{j}-\delta_{1}\right)$,

where constant $z_{G}$ is the beam depth where the clinical dose of minipeak beam is at maximum, variables $T_{i}$ and $\delta_{j}$ are the range shifter thickness for step $i$ and the compensated patient depth for depth $j$, respectively. The average clinical dose within the target at depth $j, C_{j}$, is calculated by formula;

$C_{j}=\frac{\sum_{n=I_{j}}^{N}\left[\left(A_{n}-A_{n+1}\right) \sum_{i=1}^{n} w_{i} C_{G i j}\right]}{A_{I_{j}}}$,

where $A_{i}$ and $w_{i}$ are the field area and the dose weight, respectively, for step $i$. The minimum number of contributing steps, $I_{j}$, is identical to the number for the slice covering depth $j$, satisfying inequalities;

$T_{I_{j}}-T_{1} \leq \delta_{1}-\delta_{j}<T_{I_{j}+1}-T_{1}$. 
All $w_{i}$ 's are initially set to 1 and are iteratively updated in order for $C_{j}$ 's to approach the specified clinical dose $C$, by renormalizing $w_{i}$ 's by average of $C_{j} / C$ over $j$ with weights related to the dose contribution of step $i$ to depth $j$;

$$
w_{i} \rightarrow \frac{C \sum_{j=1}^{2 N+1} A_{I_{j}} C_{G i j}{ }^{2}}{\sum_{j=1}^{2 N+1} C_{j} A_{I_{j}} C_{G i j}{ }^{2}} w_{i},
$$

where the empirically determined enhancement of the dose contribution, the square in $C_{G i j}{ }^{2}$, accelerates the convergence and the iteration will be repeated until no improvement has been observed.

\section{Dose calculation}

For the simplicity of the optimization process, the step-dose weights are determined under the implicit hypothesis such that the clinical dose is an additive parameter, which 
is equivalent to the dose-averaged RBE model for mixed radiations. ${ }^{22}$ However, since the clinical dose distribution in a patient is the most important measure for a treatment plan, we strictly follow the biophysical model for the clinical dose calculation. Namely, the distributions of physical dose, dose-averaged $\alpha$, and dose-averaged $\sqrt{ } \beta$ in the patient are calculated and then converted to the clinical dose distribution in analogy with Eqs. (4) and (5). The calculations showed a slight ( $\max 2 \%$ ) decrease of the RBE in the SOBP region while there were no differences in the plateau and tail regions, compared to the dose-averaged RBE model. This is because a large LET mixture occurs only in the SOBP region.

The physical dose calculation is based on the same algorithm as that for the conventional method, the parallel broad-beam model, where the infinite focal distance is assumed and the transverse beam flow is totally ignored. The compensated patient depths are first calculated for all the calculation points with the ray-tracing technique. For each calculation point in each step, the compensated patient depth is converted to the beam depth by adding the range-shifter thickness and the offset for the materials in 
the beam line, then converted to the broad-beam physical dose with the tabulated

minipeak depth-dose function, and then multiplied by the penumbra factor given by the error function of the distance to the field boundary. The resultant physical doses from all the steps are accumulated to form the physical dose distribution. Since the amount of time-consuming ray-tracing calculation was minimized, the typical calculation time was only twice as much as that for the conventional method.

In order to verify the dose consistency between the layer-stacking and conventional methods, we took an example of a cubic target to generate a fixed SOBP with both methods, for which we naively expected essentially the same range modulation. Fig. 5 shows the resultant clinical dose, physical dose, and dose-averaged LET along the central-axis for both methods. The distributions of the layer-stacking irradiation actually agreed very well to the conventional counterparts except for the behaviors near the ends of the SOBP due to the artificial range straggling by the minipeak filter. Paganetti and Goitein reported equivalent phenomena for proton beams with various energy spreads. ${ }^{23}$ The SOBP region for the layer-stacking irradiation made a perfect match to the target, 
giving well symmetric $90 \%$ and $95 \%$ of the nominal clinical dose at the distal and proximal ends, respectively.

\section{Example calculations}

\section{Variable SOBP formation}

We took an example of a T-shaped target in a water phantom prescribed $1 \mathrm{GyE}$ with the

layer-stacking beam. In Fig. 6(a), the grayscale represents the planned clinical dose

distribution in the isocentric plane, showing the capability of the variable-SOBP

formation conformal to the given target. Fig. 6(b) shows the planned and measured

physical dose profiles at the thin and thick parts of the SOBP. The agreement between the measurement and the calculation indicates that the entire treatment system was fully functional for the layer-stacking irradiation. 


\section{Improvement of dose distribution}

We compared the layer-stacking irradiation and the conventional irradiation by applying them to the same clinical example on the treatment planning system. Figs. 7(a) and 7(b) show isodose plots for the layer-stacking beam and the conventional beam, respectively, applied to a large tumor in the bone and soft tissue region, where the superiority of the layer-stacking method is obvious by the improved matching between $90 \%$ isodose line and the target contour. Fig. 8 shows the dose histograms for the target and the patient skin that may be an organ at risk for this case, where the skin dose was significantly reduced with the layer-stacking method while the target was receiving almost the equivalent clinical dose.

\section{DISCUSSION}


There certain are sources of inaccuracies and questionable hypotheses included in the present model: In the biophysical part, the overall fragmentation processes, the use of dose-averaged LET for LQ parameters when fragmentations and straggling are present, ${ }^{24}$ the dose-independent RBE that may be against the idea of the original LQ model, the use of the radiobiological responses of the single cell line, and the validity of the clinical factor are obviously the issues to be further investigated. In the physical beam part, the parallel broad-beam model should be improved to a better algorithm, as Petti reported, ${ }^{25}$ with a better CT-based patient model, ${ }^{26}$ and the position errors should be considered in the range compensator design. ${ }^{27}$ Even though efforts to resolve some of those problems were made, ${ }^{28} 29$ they have not yet been practiced at NIRS to avoid possible confusions in the middle of the clinical studies.

The GyE dose scale should be an original dose scale rather than cobalt-Gy equivalent. However, it may be unreasonable to measure a high-LET radiation by the equivalent low-LET radiation, where expected are larger radiobiological complexities due to the indilect effects. Concequently, we needed clinical studies to optimize the treatment 
protocols, namely, the prescription doses, fraction schedules, and the ways to add

margins to the target, for individual tumors. In other words, the present treatments with HIMAC should not be too far from the ideal treatments regardless of the definition of the GyE.

When we refine the biophysical model in the future, the use of the common set of the ridge filters for various tumor sites with various fraction doses, typically $3.6 \mathrm{GyE} \times 16$ fractions and 13.2 GyE $\times 4$ fractions at present, will have to be reconsidered. It will be, however, difficult to fabricate many protocol-specific sets of ridge filters and to install them in the present HIMAC system. On the other hand, the layer-stacking method should be very adaptive to such a model change since the biological dose optimization is fully handled by the treatment planning system.

As to the clinical usability of the layer-stacking method, we can generalize the result of the simulation study for the bone and soft tissue case: There will be substantial improvements over the conventional method for large targets when the incident beam 
directions are limited. For a small target, the improvement will be naturally small and, in a multiport plan, the conventional method will be more conformal in analogy with the 3D-conformal radiotherapy. Castro et al. also made similar studies and concluded that a reduction of the number of ports would be possible with the layer-stacking method for clinically equivalent conventional multiport treatments. ${ }^{30}$ However, there will be possible mismatches between the minipeak layers when large organ motions are present, which may deteriorate the target dose uniformity. Though Bortfeld et al. found that such side effects specific to dynamic delivery technques would be relatively small, ${ }^{31}$ we do not expect the layer-stacking method to completely replace the conventional method.

The completed treatment system will provide an option for improved conformal radiotherapy without sacrificing the functionality of the conventional method and we expect a gradual expansion of the clinical cases applicable to the layer-stacking method. The layer-stacking method should not only be specific to heavy-ion radiotherapy but also be equivalently effective for proton radiotherapy, where, in addition, majority of the problems with he biophysical model will be much less significant. 


\section{CONCLUSIONS}

The treatment planning system for HIMAC has been upgraded to adapt for the layer-stacking irradiation method based on the identical biophysical model and the calculation algorithms as those for the conventional method. Therefore, the accumulated clinical experiences with HIMAC are still valid for the layer-stacking method and hence the clinical practice is straightforward.

Improvement of dose distribution is expected with the layer-stacking method, though the significance may depend on size, shape, location of the tumor, and number of ports used to treat the patient. We expect that the conventional and layer-stacking methods will coexist in a complementary manner for a variety of clinical situations and, in general, addition of the layer-stacking method to the conventional particle treatment system should be a reasonable upgrade option for improved conformal radiotherapy. 
FIG. 1. The adopted biophysical parameters for unmodulated clinical carbon beams; (a) LQ parameter $\alpha$ and (b) LQ parameter $\beta$ as a function of carbon-ion LET, and (c) the physical dose and (d) the dose-averaged LET as a function of depth, where the solid, dashed, and dotted lines are for energies 290, 350, and 400 MeV per nucleon, respectively. The doses are normalized to the depth-zero dose.

FIG. 2. Physical and clinical doses and RBE as a function of depth for the minipeak beams; (a) the physical dose with the measured data shown as markers, (b) the clinical RBE, and (c) the clinical dose, where the solid, dashed, and dotted lines are for energies 290,350 , and $400 \mathrm{MeV}$ per nucleon, respectively. The doses are normalized to the maximum physical dose.

FIG. 3. The depth distribution for a range-compensated spherical target. The smooth line represents the cross section area of the target and the solid boxes 
indicate the regions of target slices numbered from the distal side. The dashed boxes indicate the target slices corrected by the MLC constraints for the minimum field size and for the minimum SOBP width.

FIG. 4. A schematic example for SOBP formation with depth-dose curves of the individual steps appropriately weighted for the prescribed dose of $1 \mathrm{GyE}$; (a) showing the extent of the SOBP with all the steps and (b) enlarged around the beam range only with the first four steps. The dashed lines indicate the extent of the SOBP. The dotted lines indicate sample depths numbered from the distal side.

FIG. 5. A verification of equivalency between the layer-stacking and conventional irradiations; (a) clinical dose, (b) physical dose, and (c) dose-averaged LET where the solid and dashed lines show calculations for the layer-stacking and conventional irradiations, respectively, and the circles show measured data for the layer-stacking irradiation. 
FIG. 6. An example of the dose calculation for the layer-stacking irradiation for a T-shaped (in $z-x$ plane) target, where shown are (a) the clinical isodose plot in $y=0$ plane with the overlaid target contours (solid: original, dashed: with margins) and (b) the physical dose profiles at the thick part $(x=30 \mathrm{~mm})$ and at the thin part $(x=$ $-10 \mathrm{~mm})$. The smooth lines and the circles show the calculation and the measured data, respectively.

FIG. 7. Dose distribution plots for a tumor in the bone and soft tissue region; target contour and the isodose lines overlaid on the patient $\mathrm{CT}$ image for (a) the layer-stacking irradiation and (b) the conventional irradiation. The yellow line shows the target contour while the isodose lines are in colors of the corresponding dose-percentage numbers shown in the figure.

FIG. 8. Dose distribution analysis for a tumor in the bone and soft tissue region;

(a) differential dose-volume histogram for the clinical target volume and (b) 
differential dose-surface histogram for the patient skin, where the solid and dashed

lines indicate the layer-stacking and conventional irradiations, respectively.

1 Y. Hirao, H. Ogawa, S. Yamada, Y. Sato, T. Yamada, K. Sato, A. Itano, M.

Kanazawa, K. Noda, K. Kawachi, M. Endo, T. Kanai, T. Kohno, M. Sudou, S.

Minohara, A. Kitagawa, F. Soga, E. Takada, S. Watanabe, K. Endo, M. Kumada, and S.

Matsumoto, Heavy ion synchrotron for medical use-HIMAC project at NIRS-Japan,”

Nucl. Phys. A 538, 541c-550c (1992).

2 National Institute of Radiological Sciences, "Status of the clinical studies of

heavy-ion radiotherapy — through experiences of 1000 cases (in Japanese),"

Radiological Sciences 44, 282-288 (2001).

3 W. T. Chu, B. A. Ludewigt, and T. R. Renner, "Instrumentation for treatment of

cancer using proton and light-ion beams,” Rev. Sci. Instrum. 64, 2055-2122 (1993).

4 T. Kanai, K. Kawachi, H. Matsuzawa, and T. Inada, "Broad beam three-dimensional

irradiation for proton radiotherapy," Med. Phys. 10, 344-346 (1983). 
5 Y. Futami, T. Kanai, M. Fujita, H. Tomura, A. Higashi, N. Matsufuji, N. Miyahara, M. Endo, and K. Kawachi, "Broad-beam three-dimensional irradiation system for heavy-ion radiotherapy at HIMAC," Nucl. Instrum. Methods. A. 430, 143-153 (1999). 6 B. Schaffner, T. Kanai, Y. Futami, M. Shimbo, and E. Urakabe, "Ridge filter design and optimization for the broad-beam three-dimensional irradiation system for heavy-ion radiotherapy," Med. Phys. 27, 716-724 (2000).

7 T. Kanai, K. Kawachi, Y. Kumamoto, H. Ogawa, T. Yamada, H. Matsuzawa, and T. Inada, “Spot scanning system for radiotherapy,” Med. Phys. 7, 365-369 (1980).

8 E. Pedroni, R. Bacher, H. Blattmann, T. Bohringer, A. Coray, A. Lomax, S. Lin, G. Munkel, S. Scheib, U. Schneider, and A. Tourovsky, “The 200-MeV proton therapy project at the Paul Scherrer Institute: conceptual design and practical realization,” Med. Phys. 22, 37-53 (1993).

9 T. Haberer, W. Becher, D. Shardt, and G. Kraft, "Magnetic scanning system for heavy ion therapy,” Nucl. Instrum. Methods A 330, 296-305 (1993). 
10 A. Lomax, "New techniques in hadrontherapy: intensity modulated proton beams," Phys. Med. 17, 100-102 (2001).

11 E. Urakabe, T. Kanai, M. Kanazawa, A. Kitagawa, K. Noda, T. Tomitani, M. Suda, Y. Iseki, K. Hanawa, K. Satoh, M. Shimbo, H. Mizuno, Y. Hirata, Y. Futami, Y. Iwashita, and A. Noda, "Spot scanning using radioactive 11C beams for heavy-ion radiotherapy,” Jpn. J. App. Phys. 40, 2540-2548 (2001).

12 M. Kramer, O. Jakel, T. Haberer, G. Kraft, D. Schardt, and U. Weber, "Treatment planning for heavy-ion radiotherapy: physical beam model and dose optimization," Phys. Med. Biol. 45, 3299-3317 (2000).

13 M. Kramer and M. Scholz, "Treatment planning for heavy-ion radiotherapy: calculation and optimization of biologically effective dose,” Phys. Med. Biol. 45, 3319-3330 (2000).

14 O. Jakel, M. Kramer, C. P. Karger, and J. Debus, “Treatment planning for heavy-ion radiotherapy: clinical implementation and application,” Phys. Med. Biol. 46, 1101-1116 (2001). 
15 M. Endo, H. Koyama-Ito, S. Minohara, H. Tomura, T. Kanai, K. Kawachi, H. Tsujii, and K. Morita, "HIPLAN-A heavy ion treatment planning system at HIMAC," J. Jpn. Soc. Ther. Radiol. Oncol. 8, 231-238 (1996).

16 T. Kanai, Y. Furusawa, K. Fukutsu, H. Itsukaichi, K. Eguchi-Kasai, and H. Ohara, "Irradiation of mixed beam and design of spread-out Bragg peak for heavy-ion radiotherapy," Radiat. Res. 147, 78-85 (1997).

17 H. Ito, S. Yamashita, I. Nishiguchi, W. J. Ka, S. Hashimoto, F. Yatagai, and T. Kanai “Carbon beam irradiation of monolayer cells," Nippon Acta Radiol. 53, 321-328 (1993).

18 T. Kanai, M. Endo, S. Minohara, N. Miyahara, H. Koyama-Ito, H. Tomura, N. Matsufuji, Y. Futami, A. Fukumura, T. Hiraoka, Y. Furusawa, K. Ando, M. Suzuki, F. Soga, and K. Kawachi, ”Biophysical characteristics of HIMAC clinical irradiation system for heavy-ion radiation therapy,” Int. J. Radiat. Oncol. Biol. Phys. 44, 201-210 (1999). 
of depth-dose distributions, cross sections and momentum loss,” Adv. Space Res. 17, 105-108 (1995).

20 M. Zaider and H. H. Rossi, “The synergistic effects of different radiations," Radiat. Res. 83, 732-739 (1980).

21 A. Lomax, "Intensity modulation methods for proton radiotherapy," Phys. Med.

Biol. 44, 185-205 (1999).

22 G. K. Y. Lam, "The survival response of a biological system to mixed radiations," Radiat. Res. 110, 232-243 (1987).

23 H. Paganetti and M. Goitein, "Radiobiological significance of beamline dependent proton energy distributions in a spread-out Bragg peak," Med. Phys. 27, 1119-1126 (2000).

24 G. Kraft, M. Scholz, and U. Bechthold, "Tumor therapy and track structure," Radiat. Environ. Biophys. 38, 229-237 (1999). 
particle radiotherapy,” Int. J. Radiat. Oncol. Biol. Phys. 35, 1049-1057 (1996).

26 B. Schaffner and E. Pedroni, "The precision of proton range calculation in proton radiotherapy treatment planning: experimental verification of the relation between CT-HU and proton stopping power," Phys. Med. Biol. 43, 1579-1592 (1998).

27 P. L. Petti, "New compensator design options for charged-particle radiotherapy," Phys. Med. Biol. 42, 1289-1300 (1997).

28 N. Matsufuji, H. Tomura, Y. Futami, H. Yamashita, A. Higashi, S. Minohara, M.

Endo, and T. Kanai, "Relationship between CT number and electron density, scatter angle and nuclear reaction for hadron-therapy treatment planning," Phys. Med. Biol. 43, $3261-3275(1998)$. and H. Yamashita, "A proton dose calculation code for treatment planning based on the pencil beam algorithm,” Jpn. J. Med. Phys. 18, 88-103 (1998). 
S. Pitluck, T. Fleming, J. Alonso, and E. Blakely, "Clinical gain from improved beam delivery systems,” Radiat. Environ. Biophys. 31, 233-240 (1992).

31 T. Bortfeld, K. Jokivarsi, M. Goitein, J. Kung, and S. B. Jiang, "Effects of intra-fraction motion on IMRT dose delivery: statistical analysis and simulation," Phys. Med. Biol. 47, 2203-2220 (2002). 
Fig. 1., Nobuyuki Kanematsu, Medical Physics
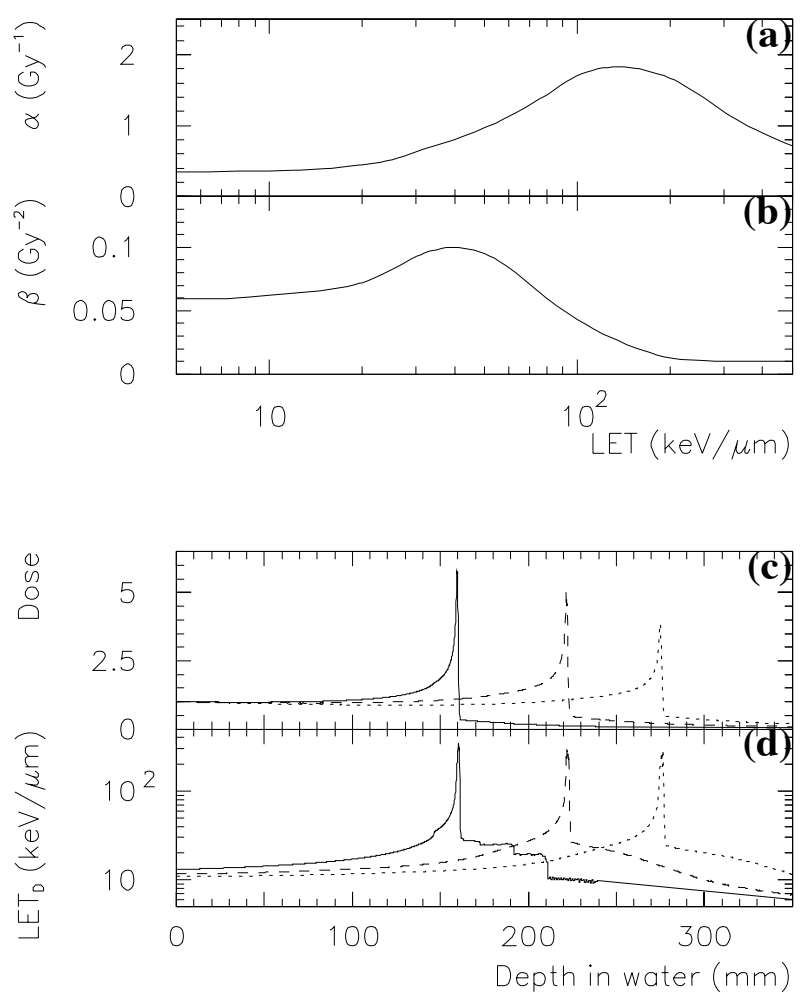
Fig. 2., Nobuyuki Kanematsu, Medical Physics

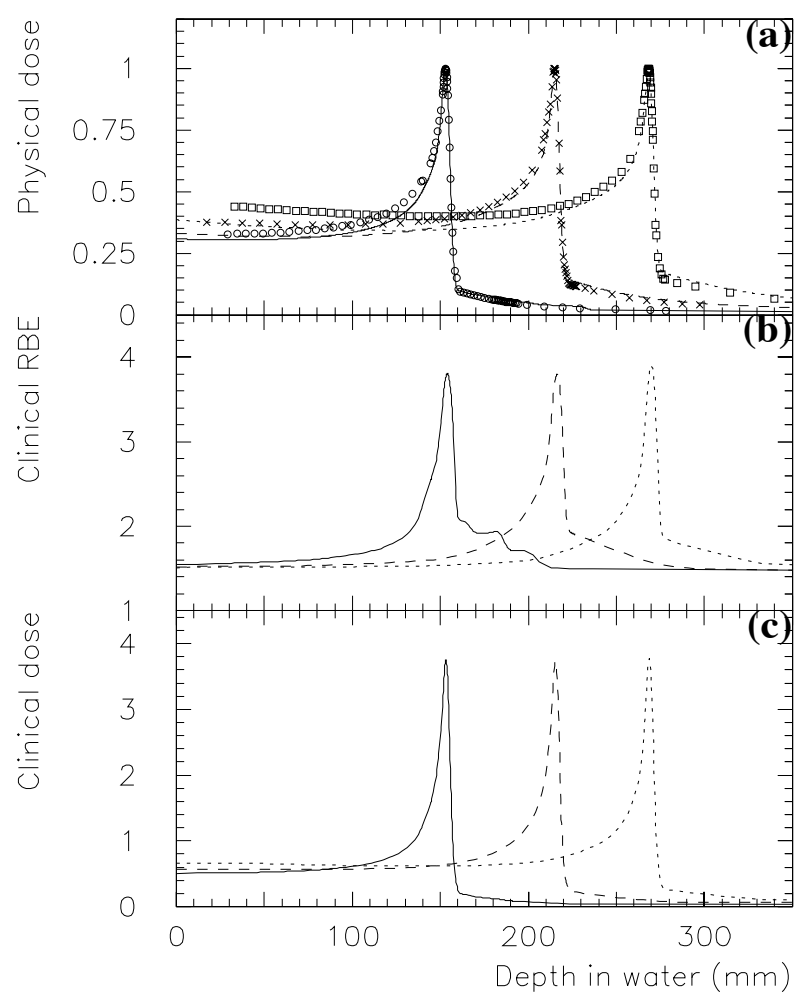


Fig. 3., Nobuyuki Kanematsu, Medical Physics

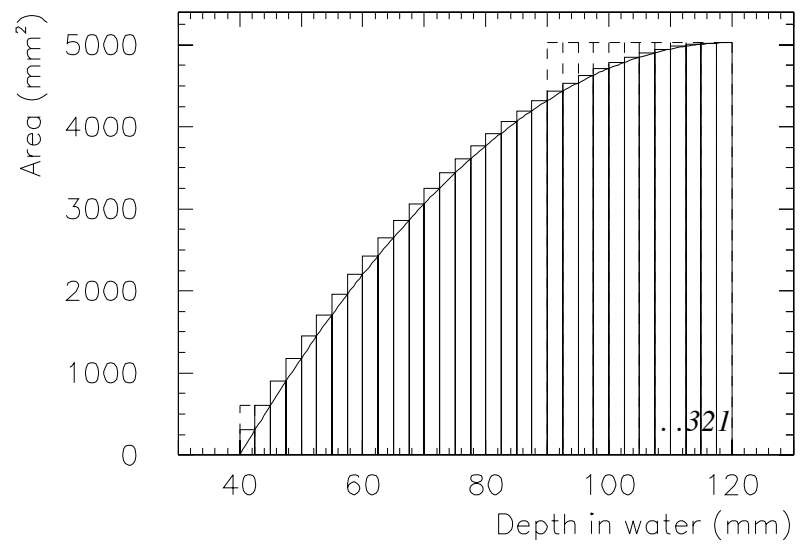


Fig. 4., Nobuyuki Kanematsu, Medical Physics
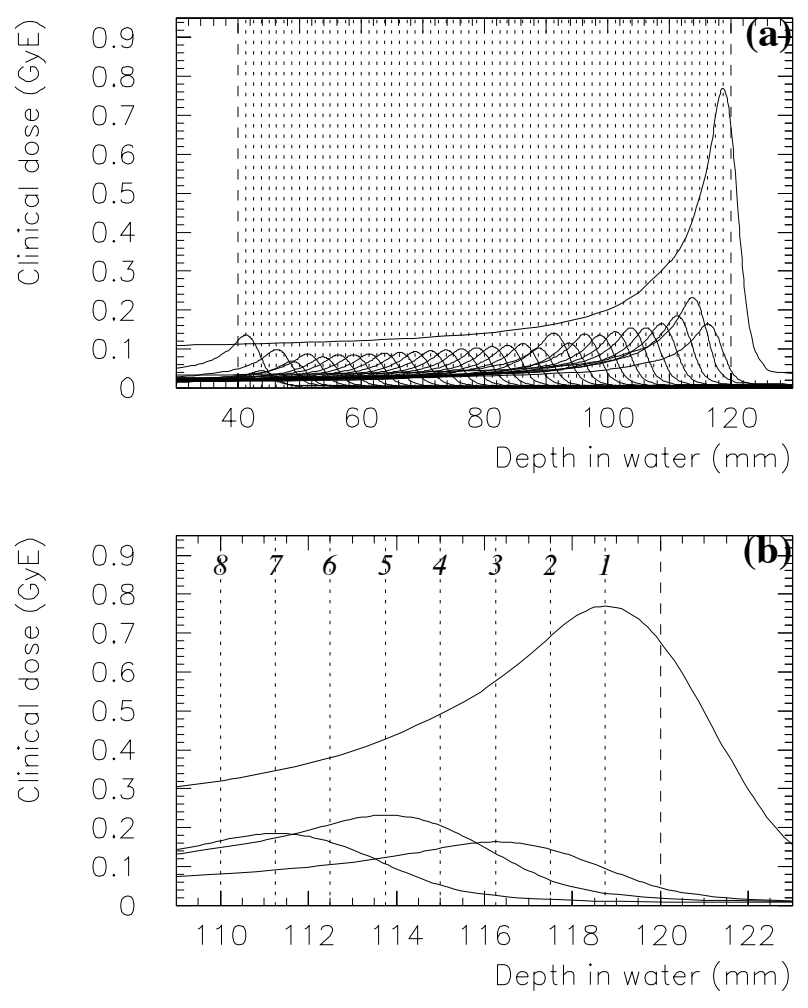
Fig. 5., Nobuyuki Kanematsu, Medical Physics

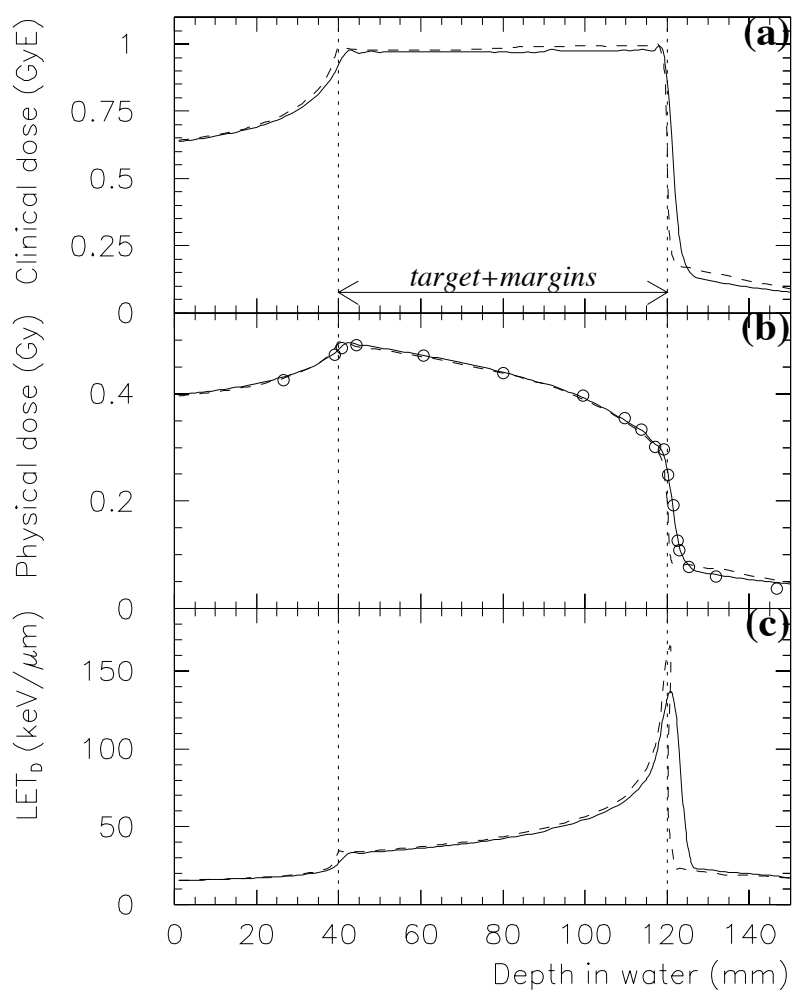


Fig. 6., Nobuyuki Kanematsu, Medical Physics
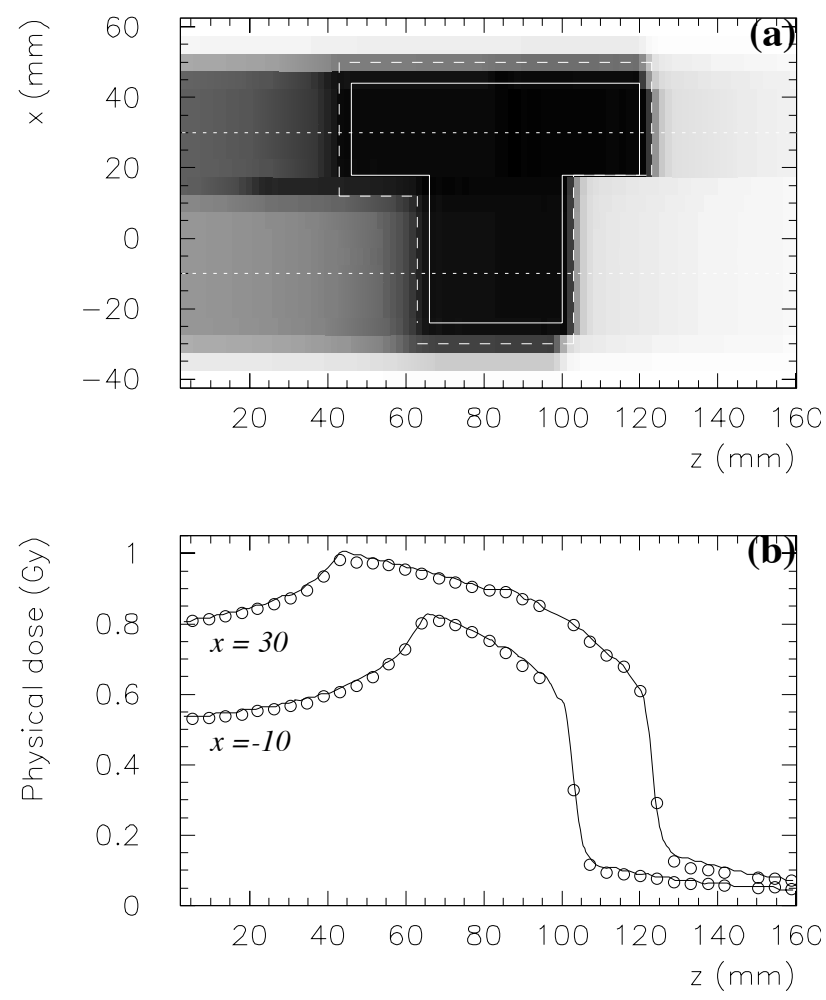
Fig. 7., Nobuyuki Kanematsu, Medical Physics

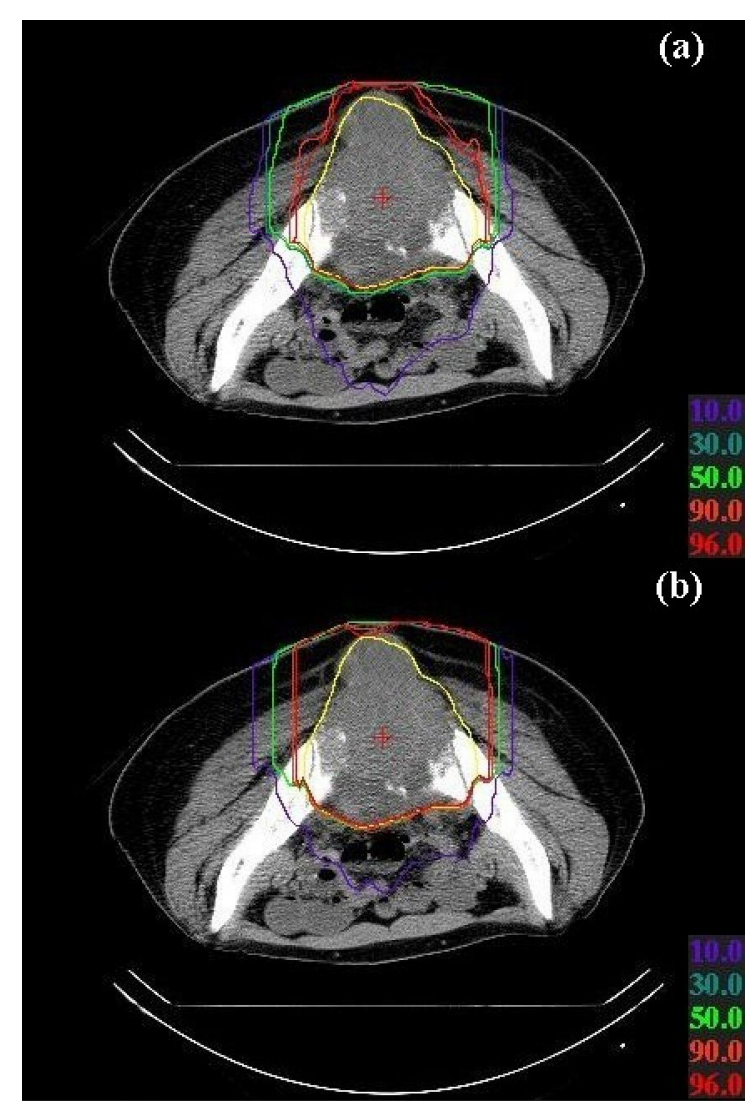


Fig. 8., Nobuyuki Kanematsu, Medical Physics
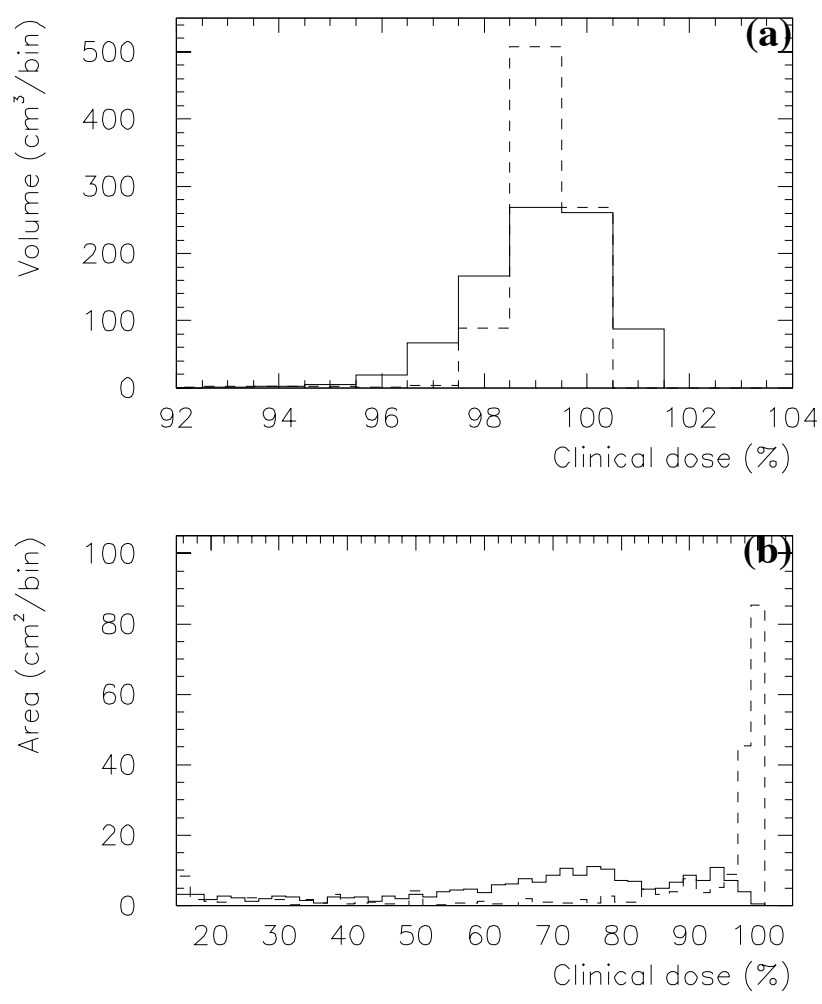